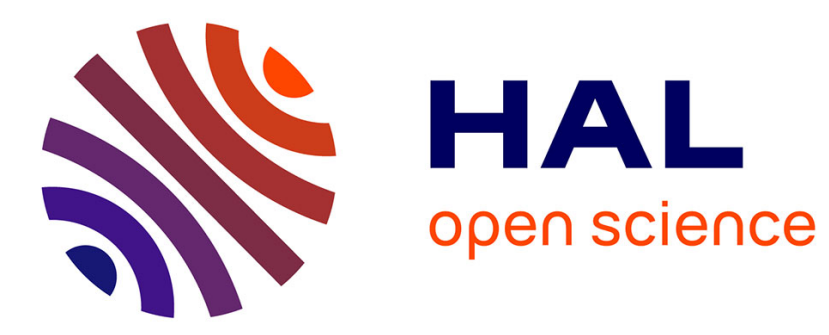

\title{
Etude et amélioration de la fiabilité d'un poromètre à diffusion utilisé en milieu naturel
}

Nader Katerji, Abdel Krim Oulid-Aïssa

\section{To cite this version:}

Nader Katerji, Abdel Krim Oulid-Aïssa. Etude et amélioration de la fiabilité d'un poromètre à diffusion utilisé en milieu naturel. Agronomie, 1983, 3 (9), pp.931-936. hal-00884592

\section{HAL Id: hal-00884592 \\ https://hal.science/hal-00884592}

Submitted on 1 Jan 1983

HAL is a multi-disciplinary open access archive for the deposit and dissemination of scientific research documents, whether they are published or not. The documents may come from teaching and research institutions in France or abroad, or from public or private research centers.
L'archive ouverte pluridisciplinaire HAL, est destinée au dépôt et à la diffusion de documents scientifiques de niveau recherche, publiés ou non, émanant des établissements d'enseignement et de recherche français ou étrangers, des laboratoires publics ou privés. 


\title{
Etude et amélioration de la fiabilité d'un poromètre à diffusion utilisé en milieu naturel
}

\author{
Nader KATERJI, Abdel Krim OULID-AÏSSA $\left({ }^{*}\right)$ \\ I.N.R.A., Station de Bioclimatologie-Télédétection, Route de Saint-Cyr, F 78000 Versailles \\ (*) I.N.A.-P.G., Laboratoire de recherche de la Chaire de Bioclimatologie, 16, rue Claude-Bernard, F 75231 \\ Paris Cedex 05
}

RÉSUMÉ A l'aide d'un dispositif simple, nous avons analysé au laboratoire le rôle du microclimat (température, vent)
sur les mesures de résistance stomatique obtenues par le poromètre à diffusion de vapeur d'eau.
Les résultats de cette étude font apparaître que le rayonnement. en provoquant des écarts thermiques entre la
chambre de mesure et la feuille testéc, conduit sclon la face à une surestimation ou à une sous-cstimation de la
valeur mesurée de résistance stomatique. Quant à la vitesse du vent, ellc conduit à une surestimation de la
valeur mesurée de résistance stomatique quelle que soit la face testée.
On a vérifié aussi que la protection de la pince grâce à une matière isolante permet de neutraliser les
perturbations induites par les facteurs climatiques.

Mot clé additionnel : Résistance stomatique.

With the help of a simple apparatus, the authors have analysed under experimental conditions the role microclimate (temperature, wind) plays on stomatal diffusion resistance measures obtained by means of a water vapour diffusion porometer. The results of this study that solar radiation, by provoking heat differences between the measuring chamber and the tested leaf, led to either an overestimation or an underestimation of the measured stomatal diffusion resistance value, depending on the leaf surface tested. Wind speed however, led to an overestimation of the measured value, no matter which surface was tested. The authors also verified that the addition of isolating material to protect the clamp had a ncutralizing effect on disturbances caused by climatic factors.

Additional key word : Stomatal diffusion resistance.

\section{INTRODUCTION}

La résistance stomatique est certainement un des paramètres les plus difficiles à obtenir en milieu naturel. Cependant, sa mesure est indispensable pour analyser le comportement hydrique d'une culture et pour modéliser la photosynthèse ou l'évapotranspiration. Une revue bibliographique de la méthodologie utilisée pour déterminer la résistance stomatique a été faite par HEATH (1959), SLAVIK (1965), KRAMER et al. (1967) et MílBURn (1979). Actuellement, l'appareillage le plus couramment utilisé est le poromètre à diffusion dont le principe a été utilisé depuis longtemps (DARWIN \& PERTZ 1911, cité par MAXIMOV, 1929). Ces appareils ont subi depuis des modifications
(HEATH \& RUSSEL, 1951 ; RASChKE, 1965 ; WEATHERLEY, 1965 ; VAN BAVEL et al., 1965 ; KANEMASU et al., 1969 ; STILES, 1970 ; MONTEITH \& BULL, 1970 ; BEARDSELl et al., 1972 ; PARKINSON \& LEGG, 1972; CAMPBELL, 1975; BINGHAM \& COYNE, 1977) qui les rendent facilement utilisables au champ, mais un certain nombre de problèmes apparaissent. En effet, póur obtenir des mesures convenables, ces appareils doivent fonctionner dans des conditions assez particulières: absence de gradient de température entre la feuille et le capteur, absence de vent favorisant les échanges hydriques entre le capteur et l'air ambiant. Or, en milieu naturel, ces conditions sont rarement réalisées. Il nous a donc paru nécessaire, d'une part, d'analyser les perturbations induites par les facteurs climatiques sur les 
mesures de résistance stomatique, d'autre part, de trouver les moyens permettant de les éviter. L'intérêt d'une telle étude paraît évident dans la mesure où elle permet de mieux cerner le problème de la signification des résultats obtenus concernant la résistance stomatique.

\section{MATÉRIEL ET DISPOSITIF EXPÉRIMENTAL}

\section{A. Poromètre à diffusion}

\section{Principe de rappareil de mesure}

Le poromètre à diffusion de vapeur d'eau utilisé (Automatic Porometer MK II) a été mis au point par MONTEITH et ses collaborateurs à l'Université de NOTTINGHAM en collaboration avec la Société DELTA-T DEVICES.

Le principe de l'appareil est le suivant: une pince contenant une petite cavité jouant le rôle de chambre de transpiration, est fixée sur la feuille, délimitant de la sorte un volume. La feuille transpire, la vapeur d'eau diffuse dans cette chambre qui contient un capteur d'humidité dont la capacité électrique varie linéairement avec l'humidité de l'air de la chambre. Après avoir fixé la pince sur la feuille, on chronomètre le temps nécessaire pour passer de l'humidité $\mathrm{H} 1$ à l'humidité $\mathrm{H} 2(\mathrm{H} 2>\mathrm{H} 1)$. Cet intervalle de temps dépend cle la facilité avec laquelle la vapeur d'cau peut s'échapper de la feuille, autrement dit, dépend de l'ouverture stomatique. L'intervalle de temps ainsi mesuré est en fait comparé à ceux obtenus, lors d'un étalonnage, avec un papier buvard humide fixé sous une plaque de polypropylène percée de trous uniformes à densité variablc. Le temps de passage de $\mathrm{H} 1$ à $\mathrm{H} 2$ est chronométré, à l'aide d'un asservissement automatique, par un compteur à vitesse constante de 25 coups/s. La mesure de résistance stomatique réduite au temps d'humidification est alors exprimée simplement par un nombre de coups.

Pour pouvoir effectuer des mesures répétitives, à la fin d'une mesure, l'humidité relative ayant atteint et même dépassée la valeur $\mathrm{H} 2$, une quantité d'air déshydraté par passage dans un tube contenant du silicagel est alors envoyée automatiquement dans la chambre de transpiration afin de la ramener à une humidité inférieure à $\mathrm{H} 1$. En général, suivant les variations entre les valeurs de résistance stomatique, 3 à 5 cycles sont nécessaires pour obtenir une valeur vraiment stable s'il n'y a pas d'autres raisons de variation. Le ternps de mesure étant faible, le constructeur de l'appareil pense que le passage d'un air sec dans la chambre de transpiration n'a pas d'effet sur la valeur de résistance stomatique obtenue; en effet, le temps d'une mesure ( $90 \mathrm{~s}$ en moyenne) est inférieur au temps d'une réaction stomatique qui est de l'ordre de 10 à $15 \mathrm{mn}$ (DJAVANChIR, 1971; de ParcevauX, 1972 ; KaneMASU \& TANNER, 1969). En cours de mesure, il faut vérifier constamment que la température dans la chambre se trouve en équilibre avec celle de la fcuille puisque d'une part, les humidité relatives $\mathrm{H} 1$ et $\mathrm{H} 2$ et, d'autre part le temps de passage de $\mathrm{H} 1$ à $\mathrm{H} 2$ en dépendent et, par conséquent, la valeur du degré d'ouverture des stomates qu'on en déduira. Pour ce faire, l'appareil est doté de 2 thermocouples permettant de contrôler la température de la feuille et de la chambre. Notons que l'expérience (PERRIER, 1968) montre que le temps d'équilibre thermique entre la feuille et la chambre (c'est-à-dire l'ensemble constitué par la pince de mesure) est inférieur (20-30 s) au temps d'une mesure.

\section{Etalonnage de l'appareil de mesure}

On mesure les temps de comptage donnés par l'appareillage utilisé avec papier buvard humidifié soit directement au contact de la chambre $\left(\mathrm{r}_{\mathrm{s}}=0\right)$, soit à l'aide de la plaquette d'étalonnage percée de trous. Sans la plaquette, on obtient un comptage correspondant seulement à la résistance à la diffusion de vapeur d'eau à l'intérieur de la chambre ; avec la plaquette en polypropylène perforée de 4 séries de trous de même diamètre et dont le nombre est variable d'une série à une autre $(11,6,4$ et 3$)$, on obtient l'équivalent de différentes valeurs de résistance stomatique. Si la résistance de chacune des séries est connue (relation 1), on peut alors établir la relation entre le nombre de coups et la résistance à la diffusion qui est, d'après le constructeur, une relation linéaire.

La résistance à la diffusion de la vapeur d'eau de chaque série de trous est donnée par l'équation suivante (MONTEITH, 1973) :

$$
r_{s}=\frac{A(L+\pi d / 8)}{n\left(\pi d^{2} / 4\right) D}
$$

$\mathrm{r}_{\mathrm{s}}$ : résistance à la diffusion de vapeur d'eau $\left(\mathrm{s} \mathrm{m}^{-1}\right)$

$A$ : surface de la chambre ouverte à l'évaporation $\left(\mathrm{m}^{2}\right)$ (m)

$\mathrm{L}$ et $\mathrm{d}$ : respectivement longueur et diamètre des trous

D: coefficient de diffusion de la vapeur d'eau, qui dépend notamment de la température $\left(\mathrm{m}^{2} \mathrm{~s}^{-1}\right)$

$\mathrm{n}$ : nombre de trous.

D'après l'équation précédente, $\mathrm{r}_{\mathrm{s}}$ dépend essentiellement du coefficient de diffusion de la vapeur d'eau, fonction de la température, ainsi que de la géométrie du système, du nombre de trous, du diamètre des trous, de l'épaisseur de la plaquette d'étalonnage et des dimensions de la chambre, grandeurs qui sont des constantes. Ainsi, si on donne à $\mathrm{D}$ sa valeur obtenue à $20^{\circ} \mathrm{C}$, on obtient les résultats suivants qui lient le nombre de trous à la résistance à la diffusion $\mathbf{r}_{\mathbf{s}}$.

\begin{tabular}{lllll}
\hline \hline $\begin{array}{l}\text { Nombre de trous } \\
\text { par série }\end{array}$ & 11 & 6 & 4 & 3 \\
\hline$r_{s}$ en s.m & & & & \\
\hline
\end{tabular}

\section{B. Dispositif expérimental}

L'analyse de l'effet du microclimat (température, vent) sur les mesures obtenues de $r_{s}$ a été effectuée en laboratoire grâce à un dispositif qui permet, dans une certaine mesure, de simuler l'action des facteurs climatiques au champ. Ce dispositif comporte 3 supports. Sur le $1^{\text {er }}$ est fixée la pince du poromètre enserrant la plaquette d'étalonnage munie d'un papier buvard humidifié ; sur le $2^{\mathrm{e}}$, mobile, est fixée une lampe à incandescence placée de telle façon que le rayonnement reçu par la pince puisse se régler à 2 intensités (600 ou $1200 \mathrm{~W} \mathrm{~m}^{-2}$ ), ce qui correspond à une plage de valeurs de rayonnement rencontrées au champ pendant une journée de beau temps; sur le $3^{\mathrm{e}}$ support est fixé un ventilateur qui permet, à l'aide d'un rhéostat, de provoquer, après étalonnage à l'aide d'un anémomètre à main, 4 vitesses de vent ( $\mathrm{V} 1=0,83 \mathrm{~ms}^{-1}, \mathrm{~V} 2=1,36 \mathrm{~ms}^{-1}$, $\left.\mathrm{V} 3=2,02 \mathrm{~ms}^{-1}, \mathrm{~V} 4=3,33 \mathrm{~ms}^{-1}\right)$. Le montage ainsi réalisé permet d'analyser le rôle d'un ou plusieurs facteurs climatiques. 


\section{RESULTATS EXPÉRIMENTAUX}

\section{A. Fiabilité du poromètre en conditions climatiques stables}

La fiabilité de l'appareil a été testée au laboratoire en effectuant une série de mesures, d'une part, sans vitesse de vent, d'autre part, sans écart de température entre le papier buvard et la chambre. Les résultats sur 26 répétitions effectuées pendant plusieurs journées dans des conditions stables de température $\left(20^{\circ} \mathrm{C}\right)$ sont présentés figure 1 .

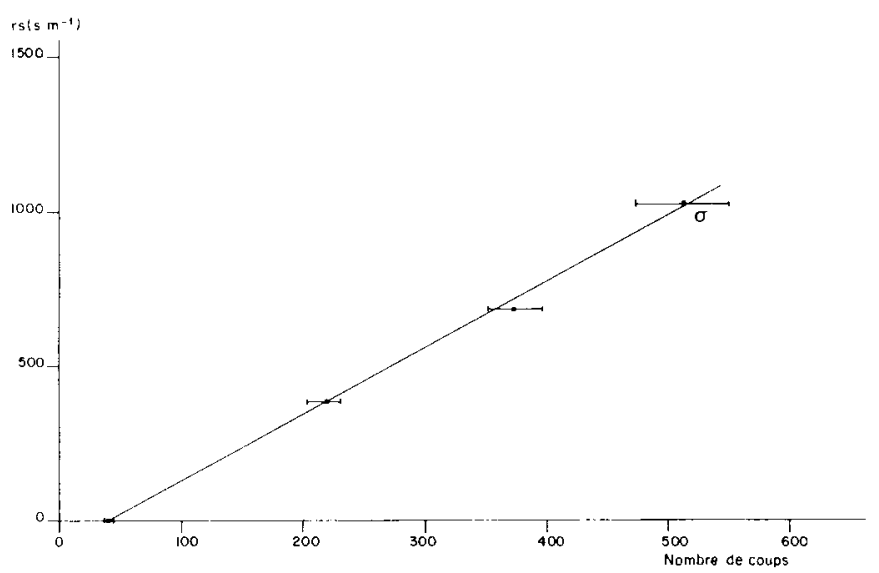

Figure 1

Courbe d'étalonnage, entre nombre de coups et résistance stomatique, établie à température constante $\left(20^{\circ} \mathrm{C}\right)$. L'écart type est calculé sur 26 répétitions.

Calibration graph of the number of counts against stomatal diffusion resistance at a constant temperature $\left(20^{\circ} \mathrm{C}\right)$. Typical variation is calculated from 26 replicates.

On constate que la fiabilité de l'appareil en conditions stables est assez satisfaisante, l'erreur sur la valeur mesurée de $r_{s}$ étant en moyenne de l'ordre de \pm 15 p. 100.

\section{B. Rôle de quelques facteurs climatiques du milieu sur l'obtention des mesures de $r_{s}$ par le poromètre}

Lorsqu'on utilise le poromètre en milieu naturel, la fiabilité de l'appareil dépend de 2 facteurs climatiques qui peuvent perturber les résultats obtenus : ce sont le rayonnement qui échauffe la chambre de transpiration, créant des écarts de température entre celle-ci et la feuille testée, et la vitesse du vent qui favorise les échanges entre la chambre et l'air ambiant si l'étanchéité n'est pas parfaite.

On a cherché à estimer le rôle respectif de ces facteurs sur les mesures obtenues de $r_{\mathrm{s}}$.

\section{Rôle de l'écart thermique}

On commence par mesurer le nombre de coups correspondant à une résistance de $375 \mathrm{sm}^{-1}$ (série de 11 trous de la plaquette d'étalonnage), de telle manière que la température de la chambre $\left(T_{c h}\right)$ soit en équilibre avec celle du papier buvard $\left(\mathrm{T}_{\mathrm{f}}\right)$. On allume la lampe dirigée sur la face supérieure, puis inférieure, de la pince de façon à provoquer un écart entre $T_{c h}$ et $T_{f}$. On constate alors que le comptage, stable au début de la manipulation, c'est-à-dire en absence de lumière (Lo), augmente ou diminue selon le cas :

si $T_{c h}>T_{f}$, le nombre de coups augmente, si $T_{c h}<T_{f}$, le nombre de coups diminue.
L'augmentation ou la diminution du nombre de coups dépend du gradient thermique. Ainsi, à titre d'exemple (fig. 2), un écart de $4{ }^{\circ} \mathrm{C}$ conduit, selon le cas, à une augmentation ou à une diminution de 100 p. 100 du comptage, ce qui pourrait conduire à des valeurs mesurées de $r_{s}$ variant du simple au double, d'après la courbe d'étalonnage présentée figure 1. A la fin de l'expérience, on vérifie que les comptages sont voisins de ceux observés en début de l'expérience (avant la perturbation), pour s'assurer que l'augmentation ou la diminution du nombre de coups traduit seulement l'effet du gradient thermique et non, par exemple, un effet parasite dû au dessèchement du papier buvard.

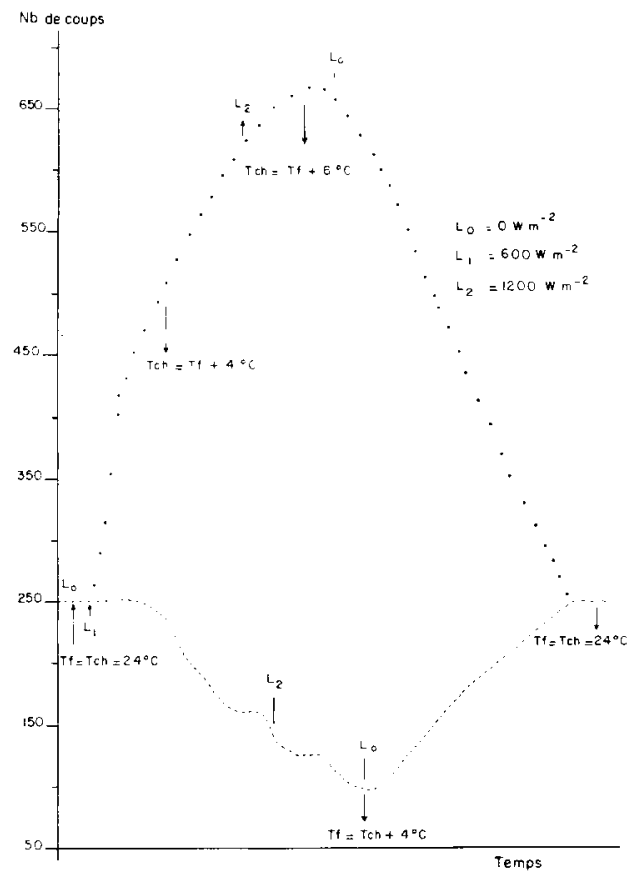

Figure 2

Influence de l'écart de température entre la chambre de transpiration $T_{c h}$ et la feuille $T_{f}$ sur le nombre de coups correspondant à une résistance stomatique de $375 \mathrm{~s} \mathrm{~m}^{-1}$.

Influence of temperature difference between the transpiration chamber $T_{c h}$ and the leaf $T_{f}$ on the number of counts corresponding to a stomatal diffusion resistance of $375 \mathrm{~s} \mathrm{~m}^{-1}$.

\section{Rôle de la vitesse du vent}

On commence par mesurer le nombre de coups correspondant aux résistances 375,690 et $1030 \mathrm{sm}^{-1}$ en absence de vent (Vo) (fig. 3). Puis, on analyse le rôle des différentes vitesses du vent sur le nombre de coups obtenus pour chacune de ces résistances. On constate alors que la vitesse du vent a un certain effet sur le comptage et que l'étanchéité de la chambre n'est pas parfaite. Cependant ce rôle est moins important que celui de la température, puisque l'augmentation du nombre de coups n'est que de l'ordre de 20 p. 100.

\section{Rôle de quelques facteurs climatiques du milieu sur les mesures de $\mathbf{r}_{\mathrm{s}}$ obtenues par une pince protégée}

Pour supprimer les effets de la température et du vent lors de l'utilisation du poromètre au champ, on a protégé la pince en fixant sur les 2 faces de minces plaquettes de matière isolante (polystyrène), puis on a testé cette protection en analysant le rôle des différentes combinaisons de 


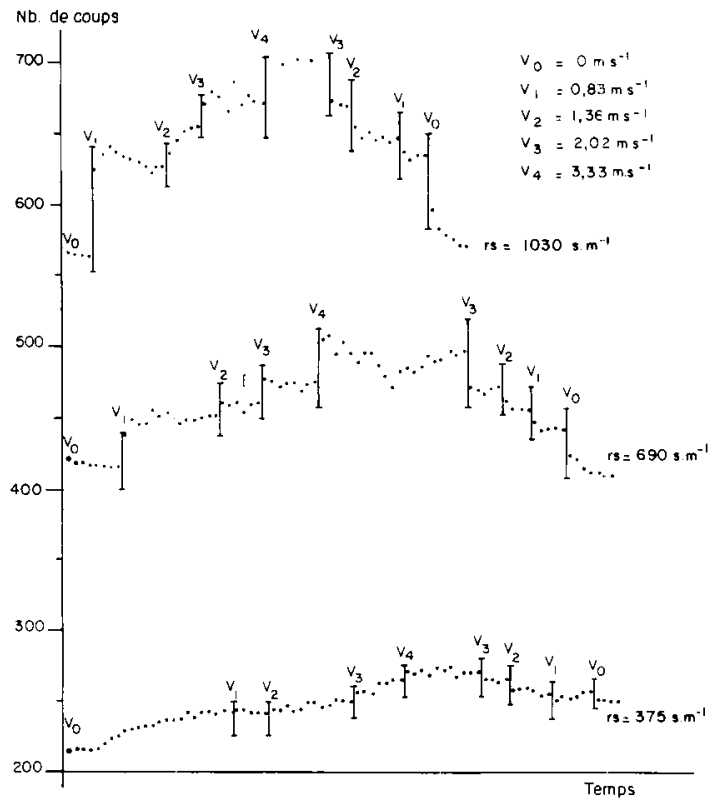

Figure 3

Influence de la vitesse du vent sur le nombre de coups correspondant aux différentes valeurs de résistance $r_{s}$.

Influence of wind speed on the number of counts corresponding to different values of resistance $r_{s}$.

lumière (celle-ci était dirigée sur la pince de façon à provoquer des valeurs de $T_{c h}$ supérieures à $T_{f}$ ) et de vent sur le comptage correspondant à une résistance de $375 \mathrm{sm}^{-1}$ en présence et en absence des facteurs climatiques indiqués.

On constate (fig. 4) que la protection de la pince neutralise bien l'influence des facteurs climatiques puisque le comptage est pratiquement identique avec ou sans perturbation. Par contre, lorsqu'on retire la protection de la pince, on observe une augmentation notable dans le comptage obtenu.

\section{CONCLUSION}

L'analyse et l'interprétation des mesures de résistance stomatique $\left(r_{s}\right)$ obtenues au champ nécessitent l'utilisation d'un appareillage qui traduise, le plus exactement possible, le phénomène de régulation stonnatique des feuilles en place.

Les résultats obtenus dans le cadre d'une étude menée au laboratoire sur la fiabilité du poromètre à diffusion (MKII)

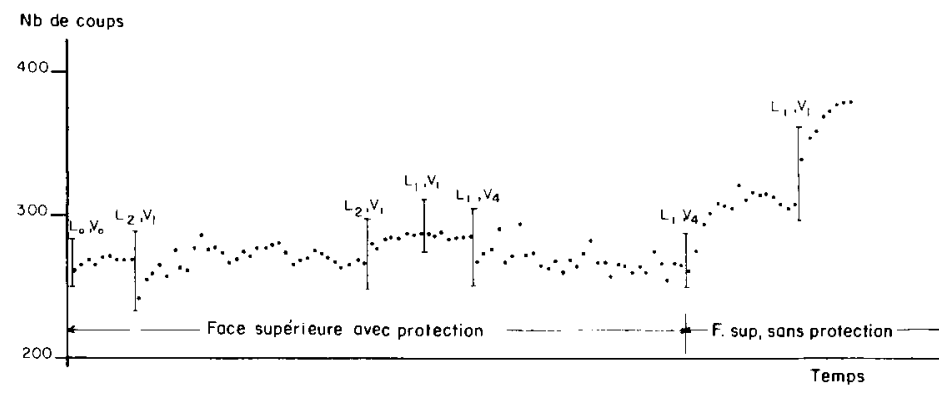

Figure 4

Influence de la vitesse du vent et de la lumière sur le nombre de coups correspondant à une résistance stomatique de $375 \mathrm{~s} \mathrm{~m}^{-1}$ en présence et en l'absence d'une protection de la pince.

Influence of wind speed and light on the number of counts corresponding to a stomatal diffusion resistance of $375 \mathrm{~s} \mathrm{~m}^{-1}$ with and without clamp protection.

montrent clairement que les mesures de $r_{s}$ obtenues au champ par cet appareil peuvent être largement entachées d'erreurs si certaines précautions, destinées à protéger la pince du poromètre, n'ont pas été prises. Ainsi, il apparaît que le rayonnement, en provoquant des écarts thermiques entre la chambre de mesure et la feuille testée, conduit, selon la face de celle-ci, à une surestimation ou à une sousestimation de la valeur mesurée de $\mathrm{r}_{\mathrm{s}}$. Quant à la vitesse du vent, elle conduit à une surestimation de la valeur mesurée de $r_{s}$, quelle que soit la face de feuille testée.

Il est intéressant de signaler que la protection de la pince à l'aide d'une matière isolante permet de neutraliser, comme on a pu le constater au cours de notre étude, l'effet des perturbations induites par les facteurs climatiques. Cette protection s'avère indispensable si on cherche à obtenir des mesures de $r_{s}$ proches de la réalité et non une simple approximation de l'ouverture stomatique.

Les résultats concernant la fiabilité du poromètre se rapportent à des expériences effectuées sur un seul type d'appareillage (poromètre à diffusion MKII), mais les conclusions tirées de notre étude restent valables pour tous les appareillages fondés sur le même principe de fonctionnement (ex. les poromètres LI-COR 1600 et 700). En effet, nous avons constaté qu'aucun système de protection de la pince de ces appareils n'est prévu lors de l'utilisation au champ.

Reçu le 17 janvier 1983. Accepté le 10 mai 1983.

\section{RÉFÉRENCES BIBLIOGRAPHIQUES}

Beardsell M. F., Jarvis P. G., Davidson B., 1972. A null-balance diffusion porometer suitable for use with leaves of many shapes. $J$. appl. Ecol., 9, 677-690.

Bingham G. E., Coyne P. I., 1977. A portable temperature controlled, steady-state porometer for field measurements of transpiration and photosynthesis. Photosynthetica, 11, (1), 148-160.

Campbell G. S., 1975. Steady-state porometer. In : « Measurement of stomatal aperture and diffusive resistance". Coll. Agr. Res. Ctr Bulletin, 809, 20-23. Washington State Univ. Pullman, Wash.

Djavanchir A., 1971. Mise au point d'une chambre de transpiration et son application à l'étude de la régulation stomatique. Thèse Doc. Ing. Univ. Orsay. $52 \mathrm{p}$. + fig.

Heath O. V. S., 1959. The water relations of stomatal cells and the mechanisms of stomatal movement.

In : "Plant physiology ", Steward F. C. éd. Academic Press New York, 193-250.

Heath O. V.S., Russel M. B., 1951. The Wheatstone bridge porometer. $J$. exp. Bot. 2, 111-116.
Kramer P. J., Didulph O., Nakayama F. S., 1967. Water absorption, conduction and transpiration. In : "Irrigation of agricultural lands", Hagan et al. éd., Agronomy n ${ }^{\circ} 11$, Amer. Soc. Agronomy, 320-336.

Kanemasu E. T., Tanner C. B., 1969. Stomatal diffusion resistance of snap beans. a) effect of light ; b) the influence of leaf water potential. Plant Physiol., 44, 1542-1546, 1547-1552.

Kanemasu E. T., Thurtell G. W., Tanner C. B., 1969. Design, calibration and field use of a stomatal diffusion porometer. Plant Physiol., 44, 881-885.

Maximov N. A., 1929. The plant in relation to water. A study of the physiological basis of drought resistance. Transl. by R. H. Yapp, George Allen and Unwiss. Ld London. $451 \mathrm{p}$.

Milburn J. A., 1979. Water flow in plants. Longmann éd. London, $225 \mathrm{p}$. 
Monteith J. L., Bull T. A., 1970. A diffusive resistance porometer for field usc. II. Theory, calibration and performancc. J. appl. Ecol., 7, 623-638.

Monteith J. L., 1973. Principles of environmental physics. Arnold éd. London, $241 \mathrm{p}$.

Parcevaux S. de, 1972. Contribution à l'étude des échanges gazeux au niveau des feuilles. Rôle des résistances ou longueurs de diffusion en écophysiologie. Thèse Doc. d'état, Univ. Orsay, 86 p. + annexes.

Parkinson K. L., Legg B. J. 1972. A continuous flow porometer. $J$. appl. Ecol., 9, 669-675.

Perrier A. 1968. Contribution à l'étude des échanges thermiques en biologie végétale. Emploi du photothermomètre de surface pour la détermination du bilan énergétique des feuilles. Rev. gén. Therm., 79/80, 721-740.
Raschke K., 1965. Suitability and construction of recording porometers for studies in stomatal physiology. Planta, 66, 113-120; 67, 225-241.

Slavik B., 1965. Water stress in plants. Czechoslovak Acad. Sci., Junk, The Hague, $322 \mathrm{p}$.

Stiles W., 1970. A diffusive resistance porometer for field use. I. Construction. J. appl. Ecol., 7, 617-622.

Van Bavel C. H. M., Nakayama F. S., Ehrler W. L., 1965. Measuring transpiration resistance of leaves. Plant Physiol., 40, 535-540. Weatherley P. E., 1965. A porometer for use in the field. The new Phytol., 66, 376-387. 